\title{
Simulating Radio Emission from Low-mass Stars
}

\author{
Joe Llama ${ }^{1}$ (D), Moira M. Jardine ${ }^{2}$, Kenneth Wood $^{2}$, Gregg Hallinan ${ }^{3}$, and Julien Morin ${ }^{4}$ (D) \\ ${ }^{1}$ Lowell Observatory, 1400 West Mars Hill Road, Flagstaff, AZ 86001, USA; joe.1lama@lowell.edu \\ ${ }^{2}$ SUPA, School of Physics \& Astronomy, University of St Andrews, North Haugh, St Andrews, Fife KY16 9SS, UK \\ ${ }^{3}$ California Institute of Technology, 1200 East California Boulevard, Pasadena, CA 91125, USA \\ ${ }^{4}$ LUPM, Université de Montpellier, CNRS, place E. Bataillon, F-34095, Montpellier, France \\ Received 2017 August 29; revised 2017 December 12; accepted 2018 January 3; published 2018 February 6
}

\begin{abstract}
Understanding the origins of stellar radio emission can provide invaluable insight into the strength and geometry of stellar magnetic fields and the resultant space weather environment experienced by exoplanets. Here, we present the first model capable of predicting radio emission through the electron cyclotron maser instability using observed stellar magnetic maps of low-mass stars. We determine the structure of the coronal magnetic field and plasma using spectropolarimetric observations of the surface magnetic fields and the X-ray emission measure. We then model the emission of photons from the locations within the corona that satisfy the conditions for electron cyclotron maser emission. Our model predicts the frequency and intensity of radio photons from within the stellar corona. We have benchmarked our model against the low-mass star V374 Peg. This star has both radio observations from the Very Large Array and a nearly simultaneous magnetic map. Using our model we are able to fit the radio observations of V374 Peg, providing additional evidence that the radio emission observed from low-mass stars may originate from the electron cyclotron maser instability. Our model can now be extended to all stars with observed magnetic maps to predict the expected frequency and variability of stellar radio emission in an effort to understand and guide future radio observations of low-mass stars.
\end{abstract}

Key words: stars: activity - stars: individual (V374 Peg) - stars: low-mass - stars: magnetic field

\section{Introduction}

One of the primary drivers in determining the space weather environment of a close-in exoplanet is the stellar magnetic field and wind. For planets orbiting $\mathrm{M}$ stars this is of critical importance when considering their potential for habitability. Due to their lower mass $\left(0.1-0.6 M_{\odot}\right)$, these stars are less luminous than solar-type stars, which in turn means the habitable zone is located much nearer to the star at a distance of $\sim 0.1-0.4$ au (Kopparapu et al. 2013). This distance makes it easier for us to detect planets orbiting within the habitable zone; however, these planets may be subjected to more frequent and intense space weather conditions than any of the planets in our solar system (e.g., Khodachenko et al. 2007; Vidotto et al. 2013; Cohen et al. 2014; See et al. 2014; Cohen et al. 2015; Garraffo et al. 2017).

In the solar system, the auroral regions of magnetized planets emit coherent, bright, polarized, low-frequency radio emission through the electron cyclotron maser instability (ECM; Zarka 1998; Farrell et al. 1999; Ergun et al. 2000; Treumann 2006; Hallinan et al. 2013) where electrons are accelerated along the planet's magnetic field lines. The power of this emission has been shown to scale directly with the incident power of the solar wind that interacts with the magnetospheric cross section of the planet. This relation, known as the "Radio Bode's law" spans many orders of magnitude in the solar system planets (Farrell et al. 1999; Zarka et al. 2001).

With over 3000 exoplanets discovered to date ${ }^{5}$ there has been considerable effort to detect radio emission from these planets. A successful detection would allow us to directly measure the magnetic field strength of the planet, which so far has only been done through indirect measurements of starplanet interactions (e.g., Shkolnik et al. 2005, 2008; Vidotto

\footnotetext{
http://www.exoplanets.org
}

et al. 2010; Llama et al. 2011; Gurdemir et al. 2012; Haswell et al. 2012). Exoplanetary magnetic fields provide insight into the internal structure and composition of the planet and potentially play a crucial role in habitability, shielding the planet from energetic particles from the stellar wind and from cosmic rays. Radio emission also offers an alternative method for directly detecting exoplanets (Farrell et al. 1999).

Extrapolations of Bode's law to exoplanets have suggested that due to their small orbital separations, hot Jupiters should emit radio emission at levels orders of magnitude greater than Jupiter in our solar system (Lazio et al. 2004). The promise of bright radio emission from exoplanets has prompted many searches; however, these have mostly yielded null detections (Ryabov et al. 2004; Lazio \& Farrell 2007; Hallinan et al. 2013). A search for the secondary eclipse of the transiting planet HD 189733 b by Smith et al. (2009) provided an upper limit at $307-347 \mathrm{MHz}$, while observations of the HAT-P-11 system by Lecavelier des Etangs et al. (2013) found a tentative detection of $150 \mathrm{MHz}$ emission from HAT-P-11b. An extensive $150 \mathrm{MHz}$ survey by Sirothia et al. (2014) found null detections from the $61 \mathrm{Vir}$ system, which was predicted to be radio-bright and also the $55 \mathrm{Cnc}$ system. At $1.4 \mathrm{GHz}$, Sirothia et al. (2014) made a tentative detection from the planetharboring pulsar PSR B1620-26, WASP-77 A b, and HD $43197 \mathrm{~b}$. A recent 2-4 and 4-8 GHz search for radio emission from $\epsilon$ Eridani b was carried out by Bastian et al. (2017); however, they could not definitively determine whether the source of the observed radio emission was from the planet.

The lack of a detection of radio emission from exoplanets is likely due to these surveys being less sensitive to the frequencies predicted from Bode's law (Farrell et al. 1999; Bastian et al. 2000; Jardine \& Collier Cameron 2008; Lanza 2009; Lazio et al. 2009; Vidotto et al. 2012; Lazio et al. 2016). Since the radio flux scales directly with the power 
of the incident stellar wind, targeting young systems that host dense, strong stellar winds may offer an exciting opportunity to make a definitive detection of radio emission from exoplanets. Indeed, there are now a number of planets known around young stars, including CI Tau b (Johns-Krull et al. 2016), V830 Tau b (Donati et al. 2016), K2-33 b (David et al. 2016; Mann et al. 2016), and TAP 26 b (Yu et al. 2017). Vidotto \& Donati (2017) carried out a theoretical study to predict the radio emission from V830 Tau b, a $\sim 2$ Myr old hot Jupiter orbiting a pre-main sequence star. By simulating the stellar wind of V830 Tau using three-dimensional MHD models coupled with magnetic imaging of the host star, these authors estimated the radio flux density from V830 Tau b to be 6-24 mJy.

Low-mass stars with spectral types later than $\sim \mathrm{M} 4$ are fully convective, meaning they lack a radiative core and a tachocline (the interface layer between the radiative core and the convective outer envelope). At even lower masses, the ultracool dwarfs (UCDs) with a spectral type $\geqslant \mathrm{M} 7$ that populate the very end of the main sequence represent a change in magnetic activity. These objects are of particular interest because they span the boundary between stars and hot Jupiters. $X$-ray observations have shown that the bolometric levels of $\mathrm{X}$-ray emission, $L_{\mathrm{X}} / L_{\mathrm{bol}}$, decrease by two orders of magnitude, suggesting they do not host a magnetically heated corona (Mohanty et al. 2002; Stelzer et al. 2006; Reiners \& Basri 2008; Berger et al. 2010). Despite the lack of X-ray emission, radio observations have revealed strong emission for UCDs spanning late $\mathrm{M}$ through $\mathrm{T}$ dwarfs, suggesting that these stars are capable of maintaining strong magnetic fields (Hallinan et al. 2008; Berger et al. 2010; McLean et al. 2011; Williams et al. 2013, 2017; Route \& Wolszczan 2016).

Radio observations of LSRJ1835+3259, an M8.5 star with a $2.84 \mathrm{hr}$ rotation period found pulsed radio emission that also phased with their simultaneous optical Balmer observations (Hallinan et al. 2015). From the frequency of this emission, Hallinan et al. (2015) were able to determine that the star hosts a magnetic field between $B \sim 1550-2850$ Gauss. Both the pulses and also the background emission from UCDs have been attributed to ECM emission (Hallinan et al. 2006, 2008). This instability is also believed to power the "stellar auroral emission" seen in the massive star CU Vir (Trigilio et al. 2004; Leto et al. 2006, 2016).

Our understanding of how the dynamo magnetic field in fully convective, low-mass stars is generated is far from complete; however, magnetic imaging of bright, rapidly rotating stars through Zeeman Doppler Imaging (ZDI; Semel 1989; Donati et al. 1997, 2006) is allowing us to study the topology and evolution of stellar magnetic fields for a wide range of stars, such as the pre-main sequence and main sequence through surveys such as BCool (solar-type stars; Marsden et al. 2014), MAPP (classical T Tauri stars; Donati et al. 2012), MiMeS (massive stars; Wade et al. 2016), MaTYSSE (young planet hosting stars; Donati et al. 2014), and BinaMIcS (short-period binary stars; Alecian et al. 2015, 2016). To map the full magnetic topology of a star, polarized spectra are collected during at least one rotation of the star. The technique is therefore most suitable for stars with rapid rotation periods. ZDI observations of low-mass stars have revealed that M0-M4 stars have weak large-scale magnetic fields, while stars later than M4 host large-scale fields that may be either strong and axisymmetric or weak and complex (Morin et al. 2008b).
One low-mass star that sits right on the boundary of being fully convective is V374 Peg. This low-mass $\left(M_{\star}=\right.$ $\left.0.28 M_{\odot}, r_{\star}=0.34 r_{\odot}\right)$ star is located in the nearby stellar neighborhood ( $d=8.93 \mathrm{pc}$; van Leeuwen 2007$)$ and is rapidly rotating $\left(P_{\text {rot }}=0.44\right.$ days; Morin et al. 2008a). V374 Peg has been observed over many years, and shows signs of frequent flaring and magnetic activity (e.g., Batyrshinova \& Ibragimov 2001; Korhonen et al. 2010; Vida et al. 2016). Given its proximity and rapid rotation, V374 Peg is an ideal candidate for magnetic imaging through ZDI.

Magnetic maps for V374 Peg were obtained on two epochs, first in 2005 August and September (Donati et al. 2006) and again a year later in 2006 August (Morin et al. 2008a). The magnetic topology of V374 Peg was found to be predominantly dipolar with a peak field strength of $B_{0}=1660 . \mathrm{G}$. Vidotto et al. (2011) used the ZDI maps as input into a 3D MHD model to compute the stellar wind properties of V374 Peg, finding that the star has a fast, dense wind with a ram pressure five times larger than that of the solar wind. V374 Peg is also radio-bright, exhibiting a rotationally modulated but smoothly varying component of emission, coupled with pulsed radio bursts that phase with the rotation period of the star (Hallinan et al. 2009).

In this paper we present the first model that couples stellar magnetic maps (observed and reconstructed using ZDI) with a model to predict the amplitude, variability, and frequency of ECM emission. In Section 2 we describe our model for simulating radio emission through ECM, including an overview of the potential field source surface extrapolation that enables us to compute the properties of the stellar corona from a ZDI map. In Section 3 we present the results of applying the model to (a) a simple inclined dipole magnetic field and (b) the magnetic map of the M dwarf V374 Peg. In Section 4 we compare the predicted ECM radio light curve for V374 Peg with near simultaneous data obtained from the Very Large Array (VLA) and show that our model is capable of reproducing both the variability and amplitude of the observations.

\section{The Model}

\subsection{Stellar Magnetic Field and Wind}

ZDI observations provide a topological map of the surface distribution of the large-scale stellar magnetic field. From these maps we can determine the structure of the stellar corona by applying a potential field source surface model (PFSS; Altschuler \& Newkirk 1969; Jardine et al. 2002). This approach assumes the magnetic field to be in a potential state and requires the prescription of two boundary conditions, one at the stellar surface, $r=r_{\star}$, and one at the source surface, $r=r_{\mathrm{ss}}$. The boundary condition at $r=r_{\star}$ is set to the radial component of the magnetic field obtained through ZDI. At $r=r_{\mathrm{ss}}$, the boundary condition that the magnetic field becomes purely radial, i.e., $B_{\theta}=B_{\phi}=0$ is imposed. This condition is analogous to imposing the maximum extent of the closed corona, and beyond the source surface the field is entirely open, carrying the stellar wind. While it is not possible to observe the extent of the closed corona for stars other than the Sun, dynamo simulations have shown that it likely varies with the fundamental parameters of the star (e.g., Réville et al. 2015). Here, we adopt the solar value of $r_{\mathrm{ss}}=2.5 r_{\star}$; however, we also ran simulations with $r_{\mathrm{ss}}=5 r_{\star}$ with negligible differences between those presented here. 


\subsection{Modeling the Coronal Density Structure}

From the magnetic field extrapolation we next determine the density structure of the stellar corona. We assume that the coronal plasma is in isothermal, hydrostatic balance, such that the pressure on each closed field line is given by

$$
p=p_{0} \exp \left[(m / k T) \int g_{s} d s\right],
$$

where $p_{0}$ is the plasma pressure at the base of the field line (which we set to $p_{0}=\kappa B_{0}^{2}$ ), $\kappa$ is a scaling parameter, $B_{0}$ is the field strength at the base of the field line, and $g_{s}=(\boldsymbol{g} \cdot \boldsymbol{B}) /|\boldsymbol{B}|$ is the component of the effective gravity along the field line. If along any field line the plasma pressure is greater than the magnetic pressure, we assume that the field line would have been forced open by the plasma pressure and we set the pressure to zero. This is also the value used for open field lines. Once the pressure is known, the density can be determined by assuming an ideal gas. We then carry out a Monte-Carlo radiation transfer simulation to produce a 3D model of the X-ray corona (Wood \& Reynolds 1999). We assume the emissivity scales directly with the local coronal density.

\subsection{Modeling Radio Emission}

In this work we are interested in simulating radio emission through the ECM instability. Using the formalism of Treumann (2006), the most efficient condition for ECM emission is that the local electron plasma frequency,

$$
\omega_{p}=\frac{e}{2 \pi} \sqrt{\frac{n_{e}}{m_{e} \epsilon_{0}}} \approx 9 \sqrt{n_{e}} \mathrm{kHz},
$$

should be less than the electron-cyclotron frequency,

$$
\Omega_{e}=\frac{e B}{2 \pi m_{e}} \approx 28 B \mathrm{kHz},
$$

where $n_{e}$ is the electron plasma density, and $B$ is the local magnetic field strength. The ECM mechanism is most efficient for $\omega_{p}^{2} / \Omega_{e}^{2} \ll 1$; however, it will also work (just less efficiently) under the condition $\omega_{p}^{2} / \Omega_{e}^{2}<1$. For completeness we therefore allow ECM emission from all sites in the coronal volume where

$$
\sqrt{\frac{n_{e} q^{2}}{m_{e} \epsilon_{0}}}<\frac{q B}{m_{e}} .
$$

In terms of the local variables determined by our coronal model, this can most usefully be written as

$$
\left[\frac{n_{e}}{10^{14} \mathrm{~m}^{-3}}\right]<\left(\frac{28}{9}\right)^{2}\left[\frac{B}{100 \mathrm{G}}\right]^{2}
$$

Regions of low density and high field strength are the most likely to emit. Locations in the coronal volume where Equation (5) is satisfied emit photons at the local gyrofrequency:

$$
\nu=\frac{q B}{2 \pi m} .
$$

For electrons, Equation (6) can be expressed as $\nu_{\mathrm{MHz}} \approx$ $2.8 \times B_{\text {Gauss }}$. We assume that photons are emitted into a hollow cone distribution, where the thickness of the cone is $1^{\circ}$ and the opening angle is $90^{\circ}$ (Melrose \& Dulk 1982). The number of electrons that can emit toward the observer at rotation phase $\phi$, and frequency $\nu$ is given by

$$
N(\phi, \nu)=\sum_{i} \exp \left(\frac{-\Delta \theta_{i}^{2}}{2 \sigma^{2}}\right) n_{i}(\nu) d V_{i}
$$

where $\Delta \theta_{i}$ is the angle between the magnetic field and the plane of the sky $\sigma$ is the thickness of the cone, $n_{i}(\nu)$ is the number of electrons in grid cell $i$ with frequency $\nu$ that can emit ECM photons, and $d V_{i}$ is the volume of the grid cell. We assume the star is optically thick and set all grid cells that are behind the star to zero. The polarization of the radio emission is determined by the sign of the local radial magnetic field.

\section{Results}

\subsection{Simple Case: Dipolar Magnetic Field}

Figure 1(a) shows a simulated magnetic map of a simple, inclined dipole. For this model the peak magnetic field strength of the dipole is set to $B_{0}=1000 \mathrm{G}$ and the inclination of the dipole axis, $\beta=40^{\circ}$. Overplotted are the results of applying the PFSS model (Section 2.1), with the closed field lines shown in red and the open, wind-bearing loops shown in blue. Figure 1(b) shows the X-ray-emitting corona for the inclined dipole (Section 2.2). In this simulation we have assumed a coronal temperature of $T_{\text {cor }}=5 \times 10^{6} \mathrm{~K}$, which is typical for rapidly rotating stars (Johnstone \& Güdel 2015). Figure 1(c) shows the regions of the corona that satisfy the conditions for ECM (Section 2.3), where we have color-coded the emission based on the polarity of the radio photons, which is determined by the sign of the local magnetic field, with red being positive and blue being negative. Finally, Figure 1(d) shows the radio spectrum for the inclined dipole. The two bright peaks in the spectrum that occur at longitude $90^{\circ}$ and $270^{\circ}$ correspond to times when the inclined dipole is in the plane of the sky, since the ECM emission is emitted at $90^{\circ}$ to the magnetic field line. Since the magnetic field is a dipole with a source surface, the field strength as a function of distance from the stellar surface can be expressed as

$$
B(r)=\frac{2 M \cos \theta}{r^{3}}\left(\frac{r^{3}+2 r_{\mathrm{ss}}^{3}}{r_{\star}^{3}+2 r_{\mathrm{ss}}^{3}}\right),
$$

where $M=B_{r}\left(r=r_{\star}, \theta=0\right) r_{\star}^{3} / 2$ is the dipole moment for a purely dipolar field (Jardine et al. 2002). Since the frequency of the ECM emission is directly related to the magnetic field strength, we can determine the maximum frequency of the radio emission, $\nu_{\max }=2.8 B_{0} \simeq 2.8 \mathrm{GHz}$.

Since we have computed the $\mathrm{X}$-ray and radio coronal densities, we can compare their observable light curves. Figure 1(e) shows the X-ray variability and also the radio variability (at $1.2 \mathrm{GHz}$ ) as a function of stellar longitude. The light curves clearly show that the X-ray variability is antiphased with the radio emission, with a Pearson correlation coefficient of $\rho=-0.87$. This anti-correlation occurs because of the field geometry: the radio intensity peaks when the dipole axis is in the plane of the sky for the observer and the maximum volume of the X-ray-emitting corona is eclipsed by the star. The longitudes of the peaks in the radio light curve 
a) Magnetic map and PFSS extrapolation

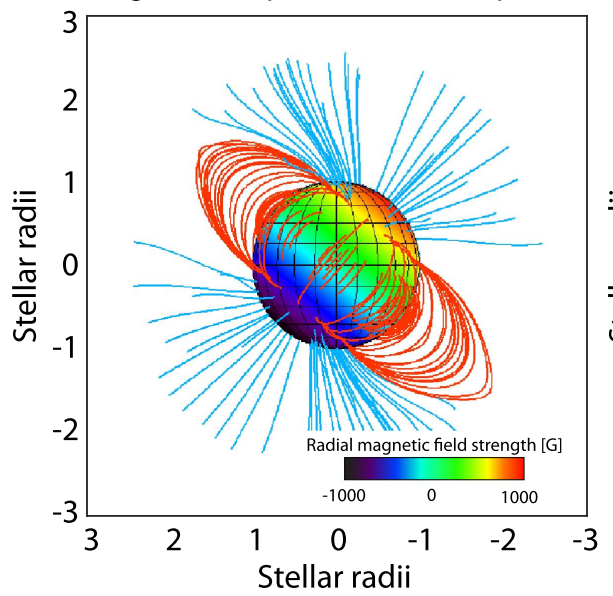

b) X-ray corona

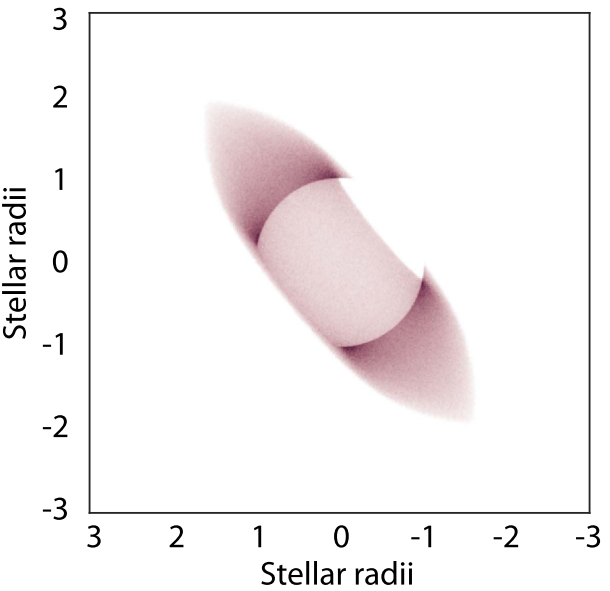

c) Radio corona

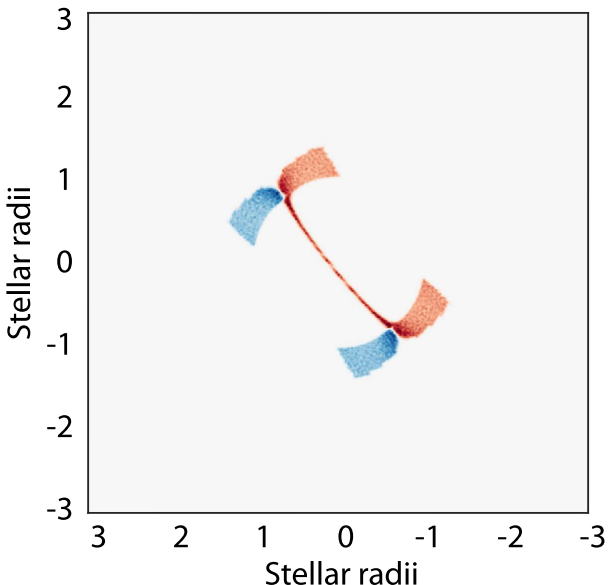

d) Radio spectrum

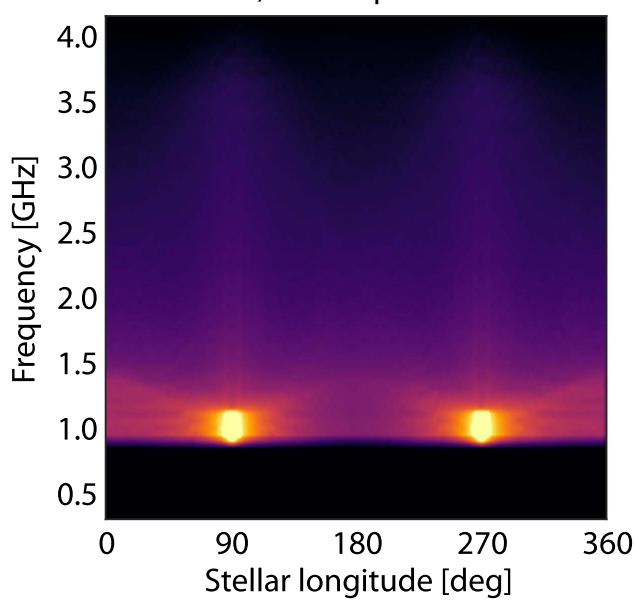

e) X-ray and radio variability

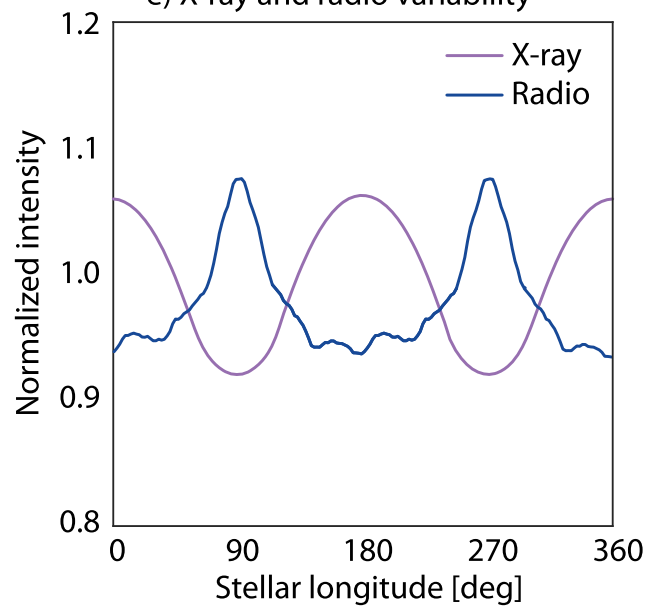

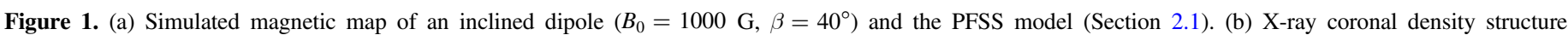

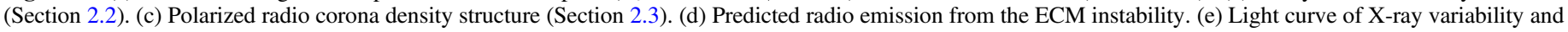

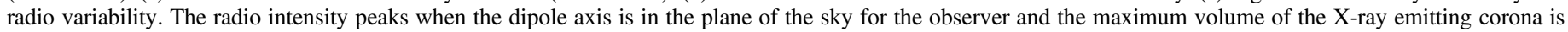
eclipsed by the star.

(Figure 1(e)) can be shown to be

$$
\phi=\arccos \left(\frac{-\tan i}{\tan (\alpha+\beta)}\right),
$$

where $i$ is the stellar inclination, $\beta$ is the angle between the magnetic and rotation axes, and $\alpha$ is the angle of the "auroral oval," which, for a dipole field is given by $\sin ^{2} \alpha=r_{\star} / r_{\mathrm{ss}}$.

\subsection{V374 Peg}

We are interested in determining the variability and frequency of radio emission that originates through the ECM instability for stars using their observed magnetic maps. Figure 2(a) shows the ZDI map of V374 Peg as reconstructed by Morin et al. (2008b) and the PFSS extrapolation. Note that the inclination of the star is such that colatitudes $\gtrsim 120^{\circ}$ are not visible as the star rotates and therefore the magnetic field cannot be reliably reconstructed in that part of the stellar disk.

Before we can compute the X-ray corona for V374 Peg we must specify the temperature of the corona, $T_{\text {cor }}$, and the value for $\kappa$, in the expression for the pressure at the base of each magnetic field line $\left(p_{0}=\kappa B_{0}^{2}\right)$. Both the coronal temperature and base pressure will alter the resultant $\mathrm{X}$-ray luminosity predicted by our model. We can therefore use observations of the X-ray luminosity to better constrain these values. X-ray observations from ROSAT of V374 Peg measured the X-ray luminosity to be $\log L_{\mathrm{X}}=28.44 \mathrm{erg} \mathrm{s}^{-1}$ (Hünsch et al. 1999). To set the temperature of the corona we use the relations derived by Johnstone \& Güdel (2015), where they show

$$
T_{\text {cor }}=0.11 \times 10^{6} F_{\mathrm{X}}^{0.26},
$$

where $T_{\text {cor }}$ is the coronal temperature in $\mathrm{MK}$ and $F_{\mathrm{X}}$ is the $\mathrm{X}$-ray flux in $\mathrm{erg} \mathrm{s}^{-1} \mathrm{~cm}^{-2}$. For V374 Peg, using the values from (Hünsch et al. 1999), we estimate a coronal temperature for V374 Peg of $T_{\text {cor }} \simeq 6 \times 10^{6} \mathrm{~K}$. Using this value of $T_{\text {cor }}$ we then varied the value of the scaling parameter, $\kappa$, to find the best fit to the observed $L_{X}$. Figure 2(b) shows the X-ray corona when our best-fit value of $\kappa$ is adopted.

Figure 2(c) shows the results of applying the model developed in Section 2.3 to determine the locations in the corona of V374 Peg that satisfy the conditions for radio emission through the ECM instability (Equation (5)). The emission is color-coded by the corresponding polarization of the emission, with red being positive and blue being negative.

While the magnetic field topology of V374 Peg is predominantly dipolar, the ZDI map does show more structure 
a) Magnetic map and PFSS extrapolation

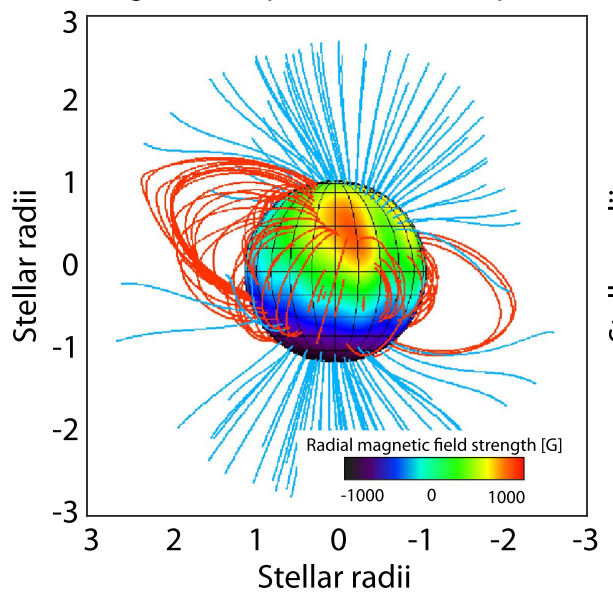

b) X-ray corona

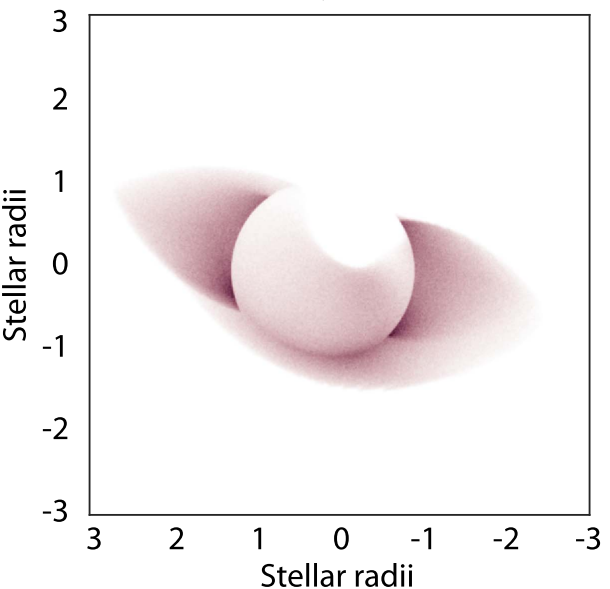

c) Radio corona

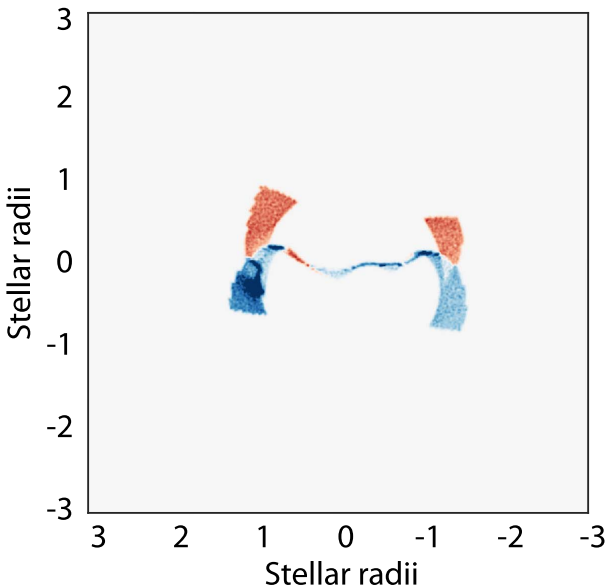

d) Radio spectrum

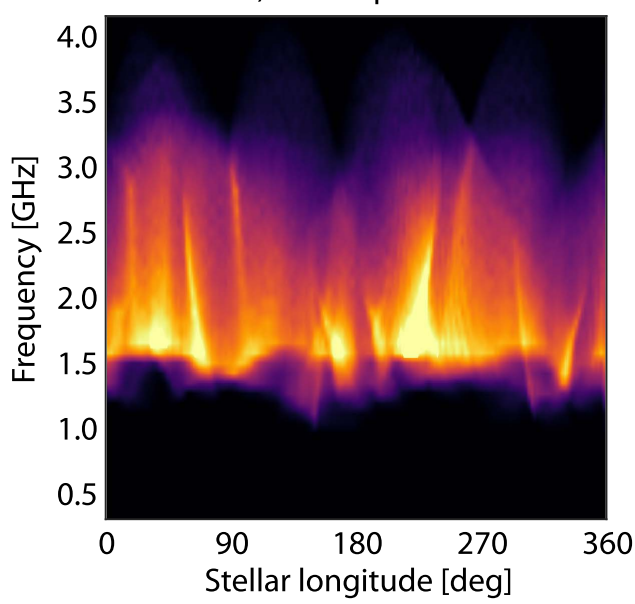

e) X-ray and radio variability

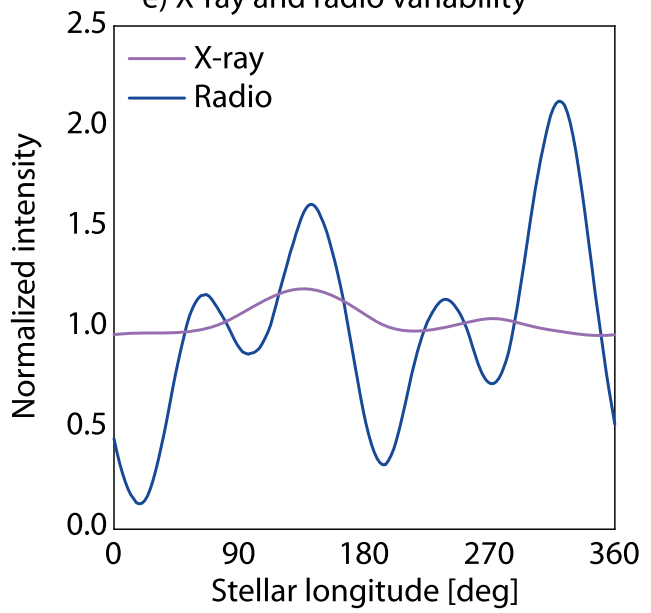

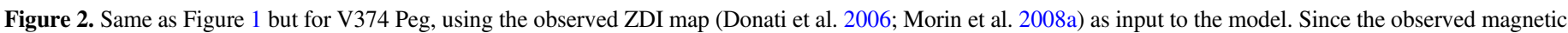
field of V374 Peg is more complex than a simple dipole, the simulated X-ray and radio coronae show more structure.

than the simple dipole shown in Figure 1. This more complex field structure manifests in a more structured X-ray and radio corona. This can be seen most clearly in the radio spectrum (Figure 2(d)) and the X-ray and radio light curves (Figure 2(e)). As with the simple dipole field, the X-ray and ECM light curves are anti-phased; however, due to the increased complexity in the magnetic field, the anti-correlation is not as strong, with a Pearson correlation coefficient of $\rho=-0.44$.

\section{Modeling the Radio Observations of V374 Peg}

V374 Peg was observed for $12 \mathrm{hr}$ on three successive nights at the VLA on 2007 January 19-21, spanning three rotations of the star (Hallinan et al. 2009). The observations were obtained using the X-band configuration of the VLA, which spans $\nu=4-8 \mathrm{GHz}$ and is therefore sensitive to a magnetic field of $B \sim 2800-4300 \mathrm{G}$. A summary of the radio observations, phased to the rotation period of V374 Peg, are shown in Figure 3 . In this light curve we have removed the pulsed radio emission and have only plotted the rotationally modulated but smoothly varying component of the radio emission, which we are attempting to model here.

These observations were taken within just a few months of the ZDI observations. If the origin of this radio emission was ECM, then our model should be able to reproduce the radio light curve. The radio spectrum shown in Figure 2 is the result of assuming every field line that satisfies the conditions for ECM does indeed emit radio photons. In reality, it is not necessarily the case that every field line is constantly emitting radio photons. To fit to the radio observations we carried out a Monte-Carlo simulation, allowing a random subset of the field lines capable of emitting ECM photons to do so. In total we ran 100,000 simulations in an effort to determine the best configuration of emitting field lines to match the VLA observations of V374 Peg.

We find that there is not a single configuration of emitting field lines that fits the observations; rather, we find many configurations that are capable of providing an equally good fit to the data. All simulations that show an equally good fit (within error) to the observations are shown as the shaded red curve in Figure 3, with the average light curve shown as the solid red line. To investigate which field lines are contributing to the phasing of the broad modulation and which contribute to the amplitude of the radio light curve, we isolated those field lines that are common to over $90 \%$ of our best-fit simulations. These field lines are shown on the PFSS extrapolations in Figure 3. We find that the common field lines are grouped into two distinct longitude regions separated by $\sim 180^{\circ}$. It is these field lines that determine the phasing of the broad modulation in the radio observations. The number of field lines that are lit, 


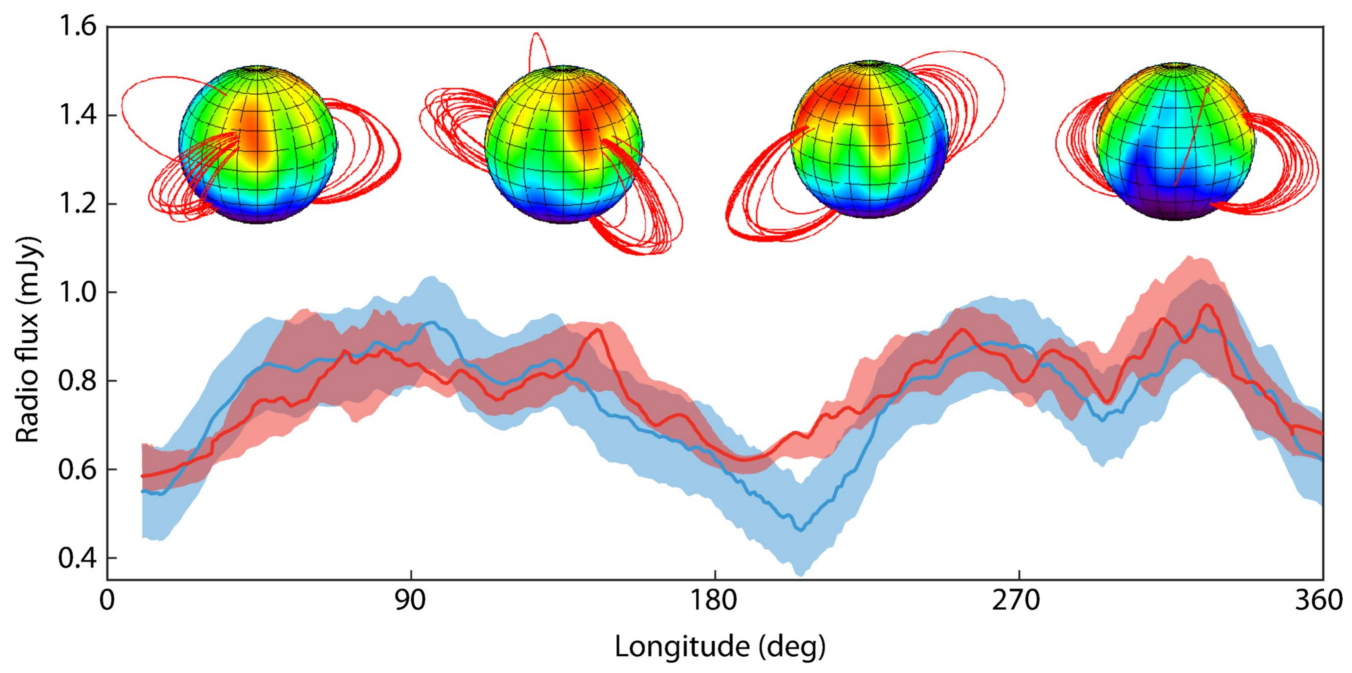

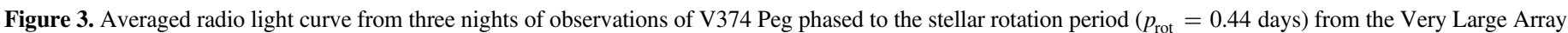

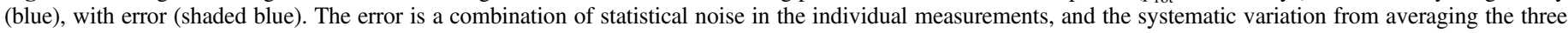

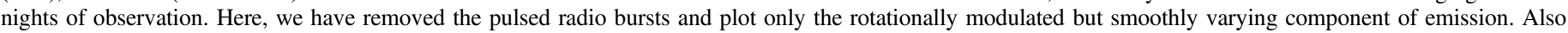

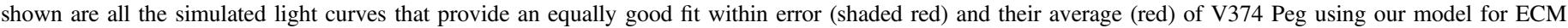

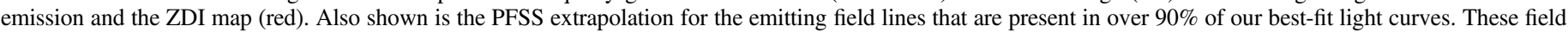
lines determine the overall phasing of the broad modulation of the radio light curve.

coupled with the choice of other field lines that are not shown in these plots, then determines the amplitude of the light curve.

To further test whether the configuration of the magnetic field determines the phasing and modulation of the radio light curve shown in Figure 3, we ran multiple simulations where we phase-shifted the observations and then found a new best fit. We found that phase-shifting the observations resulted in very poor fits to the data, and for large shifts $\left(>60^{\circ}\right)$ we were unable to find a fit at all. These additional tests suggest that the magnetic field configuration is indeed responsible for the modulation observed in the radio light curve. We also tested the role refraction may play in altering the shape of the simulated radio light curve by varying the opening angle of the emission cone from $90^{\circ}$. We found an equally good fit for opening angles $>60^{\circ}$ suggesting refraction is unlikely to be playing a critical role.

There are some caveats to our model that are worth noting. First, the magnetic map and radio observations were not obtained simultaneously, but were obtained within a few months. This is not so critical for modeling the rotationally modulated background emission since multi-epoch observations of V374 Peg have shown the magnetic field to be stable over this timescale (Donati et al. 2006; Morin et al. 2008a). However, the lack of simultaneity and the assumption in the ZDI reconstruction process that the magnetic field remains static do hinder our ability to model the pulsed radio emission. Second, the radio observations were observed in the X-band, which covers $\nu=4-8 \mathrm{GHz}(B \sim 1400-2800 \mathrm{G})$; however, in the ZDI map, the maximum magnetic field strength is $B \sim 1660 \mathrm{G}$, which means we will only simulate ECM photons at a maximum frequency of $\nu \simeq 4.6 \mathrm{GHz}$. Underestimating the magnetic flux in a ZDI map is a well-known issue and is a consequence of the reconstruction technique being less sensitive to small, strong regions of magnetic field (e.g., Lang et al. 2014).

While our model only allows for ECM emission from parts of the corona where the plasma frequency is less than the cyclotron frequency (Equation (4)), it is currently unable to account for the bursty nature of the emission and assumes steady state emission from all the ECM-capable zones. In all our simulations we find that a group of field lines is responsible for the phased modulation in the light curve (PFSS extrapolations in Figure 3). Since there are multiple field lines in these regions, our model is unable to differentiate between a single field line emitting constant levels of ECM emission, or a number of field lines emitting in a bursty fashion.

\section{Summary and Discussion}

We have developed the first model for predicting the frequency, amplitude, and rotational variability of radio emission arising through the ECM instability using realistic magnetic maps of low-mass stars obtained through ZDI. For stars that have a measurement of X-ray luminosity, our model is capable of predicting the expected frequency and rotational variability of the ECM emission.

We have benchmarked our model using ZDI observations of bright, rapidly rotating, fully convective, low-mass star V374 Peg. This star not only has magnetic maps but was also observed nearly simultaneously in the radio using the VLA. Our model successfully reproduces the amplitude and variability of the observed radio light curve, providing further evidence that the radio emission from this star could be due to the ECM instability.

We have only considered radio emission arising through the ECM instability and not through the gyrosynchrotron emission process. We use the Güdel-Benz relation to estimate the magnitude of the radio flux from gyrosynchrotron emission alone. The Güdel-Benz relation is an empirical correlation between the gyrosynchrotron radio emission and the X-ray luminosity for a wide variety of astronomical sources, including cool stars, solar flares, active galactic nuclei, and galactic black holes (Gudel et al. 1993; Guedel \& Benz 1993). The relation can be expressed as

$$
L_{\mathrm{X}} \approx L_{\nu, R} \times 10^{15.5},
$$

where $L_{\mathrm{X}}$ is the observed X-ray luminosity of the source and $L_{\nu, R}$ is the radio luminosity from gyrosynchrotron emission 
alone. Using the Güdel-Benz relation and the observed X-ray luminosity of V374 Peg $\left(L_{X}=10^{28.44}\right.$; Hünsch et al. 1999), V374 Peg's radio luminosity from gyrosynchrotron emission alone should be $L_{\nu, R}=10^{12.94}$. Using the distance to V374 Peg ( $d=8.93 \mathrm{pc}$; van Leeuwen 2007$)$, this luminosity corresponds to a radio flux of $F_{\mathrm{X}} \sim 0.08 \mathrm{mJy}$. From the VLA observations (Figure 3) the observed radio flux is at least one order of magnitude higher than this value, suggesting that gyrosynchrotron emission is a negligible contribution to the total radio flux from V374 Peg. Note that there is uncertainty in the GüdelBenz relation, particularly for low-mass stars and ultracool dwarfs that appear to lie above this relation. Simultaneous VLA and Chandra observations of the Orion Nebula Cluster by Forbrich et al. (2017) enabled these authors to search for correlations between extreme radio and X-ray variability from young stellar objects. They found 13 radio sources, all of which also exhibited X-ray variability. Multi-epoch radio, optical (including $\mathrm{H} \alpha$ ), UV (Swift), and X-ray (Chandra) observations of the UCD binary NLTT 33370 AB by Williams et al. (2015) found periodic modulation in the radio and optical and plausible modulation in $\mathrm{H} \alpha$ and the UV. Comparing simultaneous X-ray light curves with radio observations may help assess the relative contributions of radio emission through ECM and gyrosynchrotron processes. If the dominant source of radio emissions is through the ECM instability as modeled here, the radio and X-ray light curves should be anti-phased; however, if the dominant emission process is gyrosynchrotron emission then the light curves should be phased.

In the future, this model will be used to predict the expected radio emission from the ECM instability for all low-mass stars with a magnetic map and an X-ray luminosity measurement. These predictions will be useful for determining the expected frequencies at which ECM emission is likely to be observed, and will help guide future observations with the Karl G. Jansky Very Large Array. In the search for radio emission from exoplanets, our method could also potentially be used to model the stellar component to help disentangle radio signals from an orbiting exoplanet.

We would like to thank the anonymous referee for the helpful comments and suggestions. M.M.J. acknowledges support from STFC grant ST/M001296/1. This research has made use of the SIMBAD database, operated at CDS, Strasbourg, France. This research has made use of NASA's Astrophysics Data System. This research made use of Astropy, a community-developed core Python package for Astronomy (Astropy Collaboration et al. 2013).

\section{ORCID iDs}

Joe Llama (i) https://orcid.org/0000-0003-4450-0368 Julien Morin (1) https://orcid.org/0000-0002-4996-6901

\section{References}

Alecian, E., Neiner, C., Wade, G. A., et al. 2015, in IAU Symp. 307, New Windows on Massive Stars, ed. G. Meynet et al. (Cambridge: Cambridge Univ. Press), 330

Alecian, E., Tkachenko, A., Neiner, C., Folsom, C. P., \& Leroy, B. 2016, A\&A, 589, A47

Altschuler, M. D., \& Newkirk, G. 1969, SoPh, 9, 131
Astropy Collaboration, Robitaille, T. P., Tollerud, E. J., et al. 2013, A\&A, 558, A33

Bastian, T. S., Dulk, G. A., \& Leblanc, Y. 2000, ApJ, 545, 1058

Bastian, T. S., Villadsen, J., Maps, A., Hallinan, G., \& Beasley, A. J. 2017, arXiv: 1706.07012

Batyrshinova, V. M., \& Ibragimov, M. A. 2001, AstL, 27, 29

Berger, E., Basri, G., Fleming, T. A., et al. 2010, ApJ, 709, 332

Cohen, O., Drake, J. J., Glocer, A., et al. 2014, ApJ, 790, 57

Cohen, O., Ma, Y., Drake, J. J., et al. 2015, ApJ, 806, 41

David, T. J., Hillenbrand, L. A., Petigura, E. A., et al. 2016, Natur, 534, 658

Donati, J.-F., Forveille, T., Collier Cameron, A., et al. 2006, Sci, 311, 633

Donati, J.-F., Gregory, S. G., Alencar, S. H. P., et al. 2012, MNRAS, 425, 2948

Donati, J.-F., Hébrard, E., Hussain, G., et al. 2014, MNRAS, 444, 3220

Donati, J. F., Moutou, C., Malo, L., et al. 2016, Natur, 534, 662

Donati, J.-F., Semel, M., Carter, B. D., Rees, D. E., \& Collier Cameron, A. 1997, MNRAS, 291, 658

Ergun, R. E., Carlson, C. W., McFadden, J. P., et al. 2000, ApJ, 538, 456

Farrell, W. M., Desch, M. D., \& Zarka, P. 1999, JGR, 104, 14025

Forbrich, J., Reid, M. J., Menten, K. M., et al. 2017, ApJ, 844, 109

Garraffo, C., Drake, J. J., Cohen, O., Alvarado-Gomez, J. D., \& Moschou, S. P. 2017, arXiv:1706.04617

Gudel, M., Schmitt, J. H. M. M., Bookbinder, J. A., \& Fleming, T. A. 1993, ApJ, 415, 236

Guedel, M., \& Benz, A. O. 1993, ApJL, 405, L63

Gurdemir, L., Redfield, S., \& Cuntz, M. 2012, PASA, 29, 141

Hallinan, G., Antonova, A., Doyle, J. G., et al. 2006, ApJ, 653, 690

Hallinan, G., Antonova, A., Doyle, J. G., et al. 2008, ApJ, 684, 644

Hallinan, G., Doyle, G., Antonova, A., et al. 2009, in AIP Conf. Ser. 1094 15th Cambridge Workshop on Cool Stars, Stellar Systems, and the Sun, ed. E. Stempels (Melville, NY: AIP), 146

Hallinan, G., Littlefair, S. P., Cotter, G., et al. 2015, Natur, 523, 568

Hallinan, G., Sirothia, S. K., Antonova, A., et al. 2013, ApJ, 762, 34

Haswell, C. A., Fossati, L., Ayres, T., et al. 2012, ApJ, 760, 79

Hünsch, M., Schmitt, J. H. M. M., Sterzik, M. F., \& Voges, W. 1999, A\&AS, 135,319

Jardine, M., \& Collier Cameron, A. 2008, A\&A, 490, 843

Jardine, M., Collier Cameron, A., \& Donati, J.-F. 2002, MNRAS, 333, 339

Johns-Krull, C. M., McLane, J. N., Prato, L., et al. 2016, ApJ, 826, 206

Johnstone, C. P., \& Güdel, M. 2015, A\&A, 578, A129

Khodachenko, M. L., Ribas, I., Lammer, H., et al. 2007, AsBio, 7, 167

Kopparapu, R. K., Ramirez, R., Kasting, J. F., et al. 2013, ApJ, 765, 131

Korhonen, H., Vida, K., Husarik, M., et al. 2010, AN, 331, 772

Lang, P., Jardine, M., Morin, J., et al. 2014, MNRAS, 439, 2122

Lanza, A. F. 2009, A\&A, 505, 339

Lazio, J., Bastian, T., Bryden, G., et al. 2009, Magnetospheric Emissions from Extrasolar Planets, Astro2010 Science White Paper, 177

Lazio, T. J. W., \& Farrell, W. M. 2007, ApJ, 668, 1182

Lazio, T. J. W., Shkolnik, E., Hallinan, G. \& Planetary Habitability Study Team 2016, Planetary Magnetic Fields: Planetary Interiors and Habitability Technical Report, 1

Lazio, W. T. J., Farrell, W. M., Dietrick, J., et al. 2004, ApJ, 612, 511

Lecavelier des Etangs, A., Sirothia, S. K., Gopal-Krishna, \& Zarka, P. 2013, A\&A, 552, A65

Leto, P., Trigilio, C., Buemi, C. S., et al. 2016, MNRAS, 459, 1159

Leto, P., Trigilio, C., Buemi, C. S., Umana, G., \& Leone, F. 2006, A\&A, 458,831

Llama, J., Wood, K., Jardine, M., et al. 2011, MNRAS, 416, L41

Mann, A. W., Newton, E. R., Rizzuto, A. C., et al. 2016, AJ, 152, 61

Marsden, S. C., Petit, P., Jeffers, S. V., et al. 2014, MNRAS, 444, 3517

McLean, M., Berger, E., Irwin, J., Forbrich, J., \& Reiners, A. 2011, ApJ, 741, 27

Melrose, D. B., \& Dulk, G. A. 1982, ApJ, 259, 844

Mohanty, S., Basri, G., Shu, F., Allard, F., \& Chabrier, G. 2002, ApJ, 571, 469

Morin, J., Donati, J.-F., Forveille, T., et al. 2008a, MNRAS, 384, 77

Morin, J., Donati, J.-F., Petit, P., et al. 2008b, MNRAS, 390, 567

Reiners, A., \& Basri, G. 2008, ApJ, 684, 1390

Réville, V., Brun, A. S., Strugarek, A., et al. 2015, ApJ, 814, 99

Route, M., \& Wolszczan, A. 2016, ApJL, 821, L21

Ryabov, V. B., Zarka, P., \& Ryabov, B. P. 2004, P\&SS, 52, 1479

See, V., Jardine, M., Vidotto, A. A., et al. 2014, A\&A, 570, A99

Semel, M. 1989, A\&A, 225, 456

Shkolnik, E., Bohlender, D. A., Walker, G. A. H., \& Collier Cameron, A. 2008, ApJ, 676, 628

Shkolnik, E., Walker, G. A. H., Bohlender, D. A., Gu, P.-G., \& Kürster, M. 2005, ApJ, 622, 1075 
Sirothia, S. K., Lecavelier des Etangs, A., Gopal-Krishna, Kantharia, N. G., \& Ishwar-Chandra, C. H. 2014, A\&A, 562, A108

Smith, A. M. S., Collier Cameron, A., Greaves, J., et al. 2009, MNRAS, 395, 335

Stelzer, B., Micela, G., Flaccomio, E., Neuhäuser, R., \& Jayawardhana, R. 2006, A\&A, 448, 293

Treumann, R. A. 2006, A\&ARv, 13, 229

Trigilio, C., Leto, P., Umana, G., Leone, F., \& Buemi, C. S. 2004, A\&A, 418,593

van Leeuwen, F. 2007, A\&A, 474, 653

Vida, K., Kriskovics, L., Oláh, K., et al. 2016, A\&A, 590, A11

Vidotto, A. A., \& Donati, J.-F. 2017, A\&A, 602, A39

Vidotto, A. A., Fares, R., Jardine, M., et al. 2012, MNRAS, 423, 3285

Vidotto, A. A., Jardine, M., \& Helling, C. 2010, ApJL, 722, L168
Vidotto, A. A., Jardine, M., Morin, J., et al. 2013, A\&A, 557, A67

Vidotto, A. A., Jardine, M., Opher, M., Donati, J. F., \& Gombosi, T. I. 2011, MNRAS, 412, 351

Wade, G. A., Neiner, C., Alecian, E., et al. 2016, MNRAS, 456, 2

Williams, P. K. G., Berger, E., Irwin, J., Berta-Thompson, Z. K., \& Charbonneau, D. 2015, ApJ, 799, 192

Williams, P. K. G., Berger, E., \& Zauderer, B. A. 2013, ApJL, 767, L30

Williams, P. K. G., Gizis, J. E., \& Berger, E. 2017, ApJ, 834, 117

Wood, K., \& Reynolds, R. J. 1999, ApJ, 525, 799

Yu, L., Donati, J.-F., Hébrard, E. M., et al. 2017, MNRAS, 467, 1342

Zarka, P. 1998, JGR, 103, 20159

Zarka, P., Treumann, R. A., Ryabov, B. P., \& Ryabov, V. B. 2001, Ap\&SS, 277, 293 